\title{
Electronic Source Data
}

National Cancer Institute

\section{Source}

National Cancer Institute. Electronic Source Data. NCI Thesaurus. Code C142534.

Digital information in original records, and certified copies of digital information in original records. 